\title{
A systems level strategy for analyzing the cell death network: implication in exploring the apoptosis/autophagy connection
}

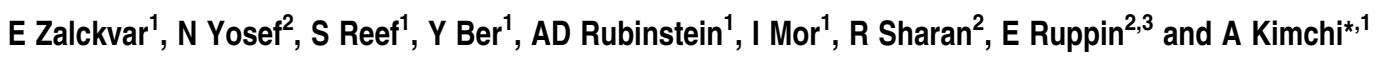

The mammalian cell death network comprises three distinct functional modules: apoptosis, autophagy and programmed necrosis. Currently, the field lacks systems level approaches to assess the extent to which the intermodular connectivity affects cell death performance. Here, we developed a platform that is based on single and double sets of RNAi-mediated perturbations targeting combinations of apoptotic and autophagic genes. The outcome of perturbations is measured both at the level of the overall cell death responses, using an unbiased quantitative reporter, and by assessing the molecular responses within the different functional modules. Epistatic analyses determine whether seemingly unrelated pairs of proteins are genetically linked. The initial running of this platform in etoposide-treated cells, using a few single and double perturbations, identified several levels of connectivity between apoptosis and autophagy. The knock down of caspase 3 turned on a switch toward autophagic cell death, which requires Atg5 or Beclin-1. In addition, a reciprocal connection between these two autophagic genes and apoptosis was identified. By applying computational tools that are based on mining the protein-protein interaction database, a novel biochemical pathway connecting between Atg5 and caspase3 is suggested. Scaling up this platform into hundreds of perturbations potentially has a wide, general scope of applicability, and will provide the basis for future modeling of the cell death network.

Cell Death and Differentiation (2010) 17, 1244-1253; doi:10.1038/cdd.2010.7; published online 12 February 2010

The process of programmed cell death (PCD) is driven by a network of proteins connected to each other in an intricate manner. Over the past two decades, many of the network's proteins (nodes) and the interactions among them (edges; mostly post-translation modifications) have been identified. The PCD network is turned on by well-defined input signals, such as activation of death receptors or exposure to DNA damaging agents. The efficiency of its performance determines the individual cell's probability to die, which, when assessed over a large population of cells, can be translated into the percent of cell death. Dying cells can display several distinct cell death phenotypes, each driven by a different subset of proteins and molecular pathways. Examples are the caspase-dependent apoptotic cell death, autophagic cell death and programmed necrosis. ${ }^{1}$ Cells exposed to the same input signal can switch from one cell death modality to another in response to specific perturbations, ${ }^{2-4}$ and in some cases, a mixed type of cell death can also be observed. ${ }^{5-7}$ This led us to propose here a working model, according to which the proteins that mediate the three different cell death phenotypes should be integrated within a common network, and the corresponding subsets of proteins should be considered as functional modules within this global network. To study the network as a whole, new strategies capable of analyzing the connectivity within and between the functional modules are required.

Here, we developed a platform for dissecting the network's architecture, which is based on single and double sets of RNAi-mediated perturbations. The uniqueness of this platform is that it combines three approaches to analyze the outcome of the perturbations including: (1) The use of an unbiased quantitative reporter (the luciferase gene) to measure the extent of cell death and calculate the fitness values of the genetic interactions between seemingly unrelated pairs of genes. (2) Assessing the perturbation effects on the molecular responses to the cell death agent, not only downstream to the knocked-down gene but also at distant functional modules. (3) Applying computational methods for the discovery of pathways mediating the newly identified genetic interactions by mining the protein-protein interaction (PPI) database. We applied this platform to cells that were exposed to the DNA damaging drug etoposide, targeting a few pairs of apoptotic and autophagic genes as a proof of concept. The initial running of this platform identified several levels of connectivity between apoptotic and autophagic proteins that either provide backup compensatory switches contributing to the robustness of the system or augment the final cell death performance through a novel non-canonical pathway.

\footnotetext{
${ }^{1}$ Department of Molecular Genetics, Weizmann Institute of Science, Rehovot 76100, Israel; ${ }^{2}$ Blavatnik, School of Computer Science, Tel-Aviv University, Tel-Aviv 69978 , Israel and ${ }^{3}$ School of Medicine, Tel-Aviv University, Tel-Aviv 69978, Israel

*Corresponding author: A Kimchi, Department of Molecular Genetics, Weizmann Institute of Science, Herzel, Rehovot 76100, Israel. Tel: + 97289342428 ;

Fax: + 9728931 5938; E-mail: adi.kimchi@weizmann.ac.il

Keywords: programmed cell death; autophagy; caspase3; protein network

Abbreviations: $\mathrm{PCD}$, programmed cell death; PPI, protein-protein interaction

Received 02.7.09; revised 30.11.09; accepted 05.1.10; Edited by G Melino; published online 12.2.10
} 


\section{Results}

Designing the general outline of the platform. Our goal was to develop a platform that would assess the effects of single and double RNAi-mediated perturbations on both the final cell death performance, and the molecular responses to the death-inducing agent. Subsequently, the nature of epistatic interactions between seemingly unrelated pairs of genes is determined by this platform, and computational methods are applied to identify the pathway that connects these pairs of proteins (see Figure 1 for scheme). The strategy is based on transient transfections with short hairpin RNAs (shRNAs) targeting genes from the different functional modules of the network $72 \mathrm{~h}$ before exposure to the deathinducing agent. HEK293 cells were chosen for this purpose because of their high sensitivity to different death-inducing agents, including etoposide (see below), and their high transfection efficiency, which enables the knock down of two proteins simultaneously. Moreover, these cells can undergo different types of PCD. ${ }^{8-10}$ For assessing the responses of control non-perturbed cells, we used shRNA targeting HcRed (Supplementary Figure S1; see also supplementary text). To exclude off-target effects, two different shRNA plasmids targeting the same gene were used. It is noted that all the individual shRNA plasmids used in this work had no effect on cell viability in non-treated cells, and therefore could be safely used for exclusively testing their possible effects on the cell death responses to the input signal.

To choose the genes to be targeted by hypothesis-driven approaches, as opposed to random knock down, we constructed an initial connectivity map that served as our working model chart (Supplementary Figure S2). The map comprises a major part of the most studied apoptotic, autophagic and programmed necrotic proteins, organized into their canonical pathways, constituting three functional modules within the network. The proteins in the map (which we defined in this work as the PCD set) are linked by associations constructed manually on the basis of published knowledge (see Supplementary Tables 1 and 2 for the list of proteins and edges, respectively).

Establishing the cellular and the molecular readouts. The development of the cell death readout required a quantitative accurate assay that is capable of quantifying all forms of cell death. To this end, we developed a multi-well formatted luciferase assay using HEK293 cells engineered to stably express the luciferase gene. After exposure to a given cell death-inducing agent, surviving cells that express luciferase are quantified by enzymatic activity, with the distinct advantage that, unlike other readouts, this assay does not measure specific characteristics of only one type of cell death. Residual cell viability is calculated by dividing the values of treated by non-treated cells, and the resulting cell death values measured in transfectants carrying control shRNA are normalized to $100 \%$ cell death performance. Assessing cell death by this assay was consistent with other more traditional and less accurate cell death assays, such as Trypan blue exclusion tests. ${ }^{11}$ It is noted that the luminescence values are linear over several orders of magnitude, providing a wide window of detection of viable cells (between $10^{2}$ and $10^{5}$ cells; Supplementary Figure S4a). Moreover, every point in each experiment is tested in 10 replicates, thus conferring a high degree of

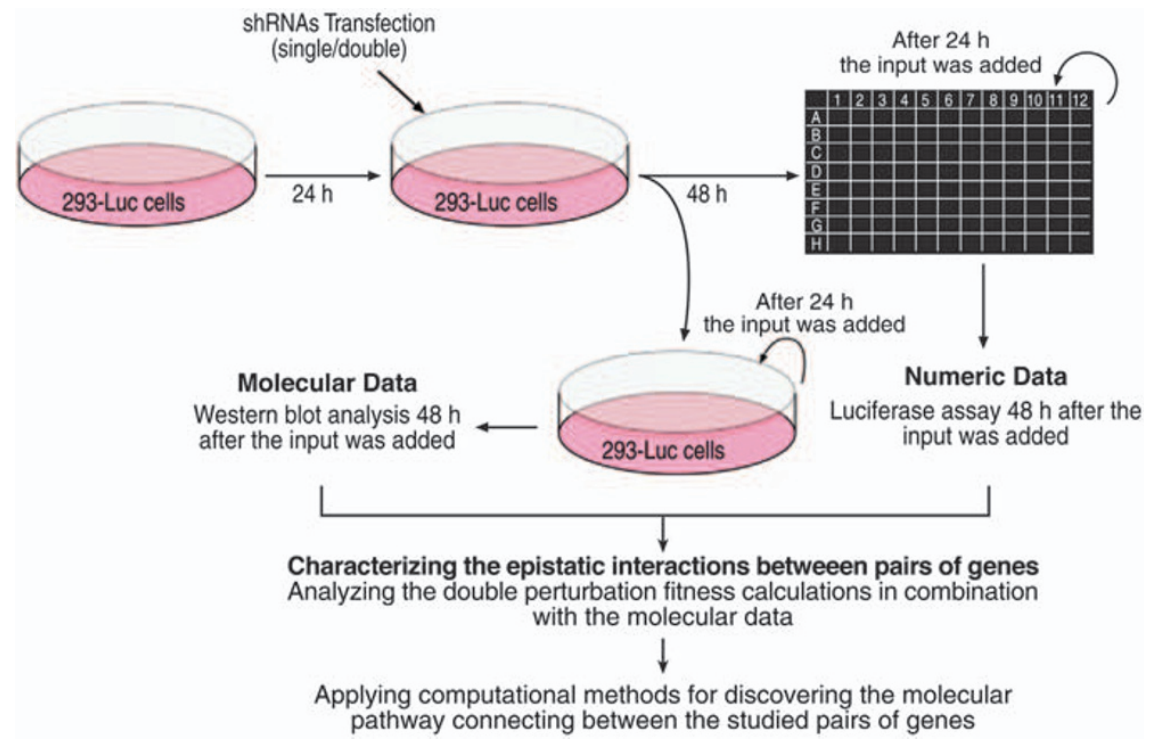

Figure 1 A platform to analyze the PCD network, which combines quantitative assessment of cell death, molecular responses, and computational approaches for novel pathway discovery. HEK293-luciferase (293-Luc) cells were transfected with shRNA targeting single or pairs of genes or with HcRed-targeting shRNA as a negative control. Two days after transfection, the cells were split into black 96 -well plates, and in parallel to $9 \mathrm{~cm}$ plates. After $24 \mathrm{~h}$, the culture media were replaced with fresh media containing $50 \mu \mathrm{M}$ etoposide (input) or DMSO as a negative control. After $48 \mathrm{~h}$, the luciferase assay was performed in 96 -well plates in ten replicates for each perturbation, and in parallel, the cells grown in the $9 \mathrm{~cm}$ plates were extracted for molecular analysis, mainly by western blot. Epistatic interactions between pairs of genes were further processed by combining the double perturbation fitness calculations from the luciferase assay with the molecular data. Computational approaches were used for the discovery of novel pathways connecting pairs of genes that display genetic interactions 


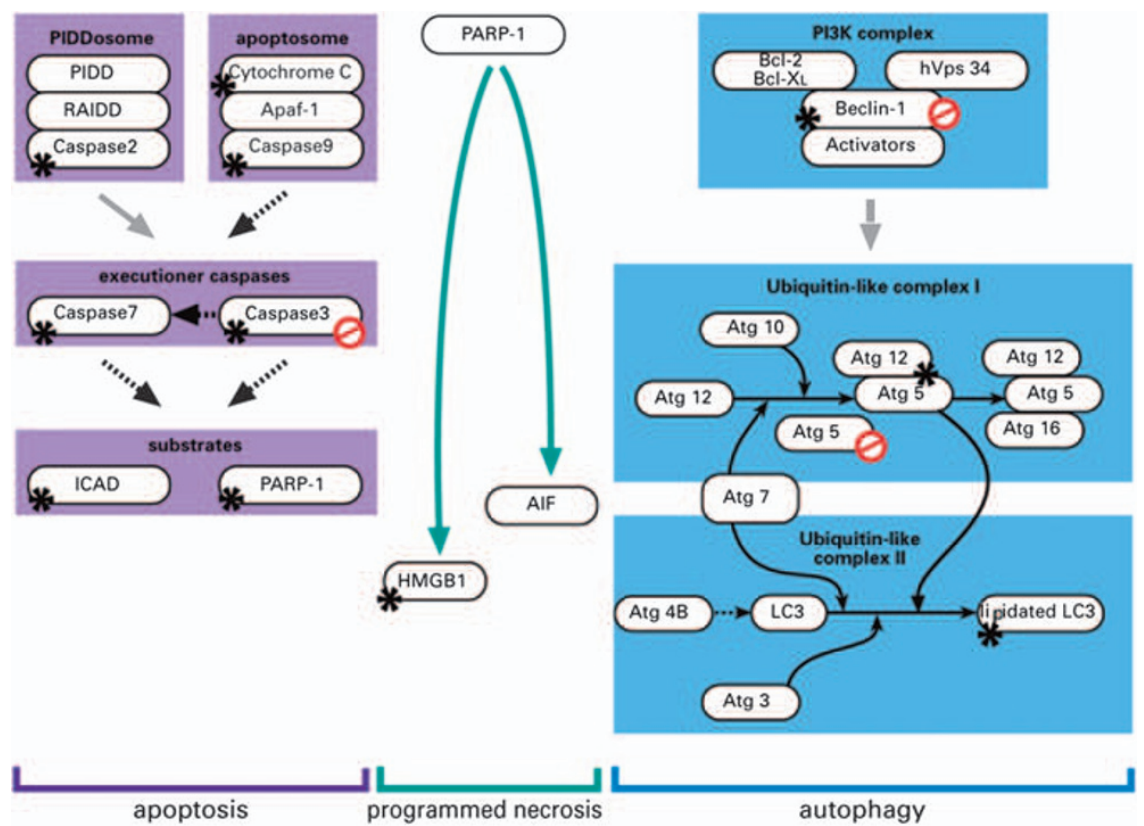

Figure 2 A scheme describing the hierarchy/connectivity among the analyzed proteins. Red signs indicate proteins that were perturbed using RNAi. Asterisks indicate proteins whose status in response to the input signal was examined as follows: the extent of the proteolytic cleavage of various proteins (caspases3, 7, 2, 9, PARP-1, ICAD), cytochrome $c$ release, assessment of the catalytic activity of PARP-1 (by measuring PAR ribosylation of proteins), activation of LC3 measured by fluorescent microscopy (assessing the punctate staining of lipidated LC3) and assessment of HMGB1 release. Dashed arrows represent proteolytic cleavage. Gray arrow indicates that the edge connectivity was confirmed genetically (by knock out/knock down strategies) without yet defining direct interactions between specific nodes

accuracy (Supplementary Figure $S 4 b$; the statistical analysis is detailed in Materials and Methods section). Thus, statistically significant small changes in cell death performance can be detected and quantified, an important advantage when considering the complexity of biological networks that tend to be robust and therefore relatively resistant to perturbations. These numeric data are then used to quantify the degree of epistasis between pairs of proteins, defining aggravating or alleviating types of interactions between seemingly unrelated genes in the network (as detailed in Materials and Methods section).

The second readout consists of testing the outcome of the perturbations on the molecular responses to the death-inducing agent, that is, measuring the status of interactions between the network's nodes. This was based on western blotting using antibodies that recognize the relevant protein modifications in the pathway. It includes antibodies that recognize the cleaved substrates of caspases, including the processing/activation of the initiator and executioner caspases, poly(ADP) ribosylation of proteins, and HMGB1 release into culture medium (marked in asterisks in Figure 2 and shown in Figures 3 and 4). Additional readouts that complement the western blotting assessments included cellular staining with molecular markers that reflect activation of specific pathways in the different modules, such as DAPI staining for tracing condensed/fragmented nuclei, cytochrome $c$ release from mitochondria or LC3 punctate staining in the cytosol, reflecting autophagic activity (see Figures 3 and 4 and Supplementary Figure S5).
Computational approaches used for novel pathway discovery. The next stage in the platform involved the discovery of the molecular pathway(s) connecting pairs of proteins that display newly identified genetic interactions. To this end, a map of human PPIs was constructed ( $\sim 40 \mathrm{~K}$ interactions connecting $\sim 10 \mathrm{~K}$ proteins) on the basis of interactions from the Human Protein Reference Database ${ }^{12}$ and from large-scale screens including yeast two hybrid ${ }^{13,14}$ and mass-spec analysis. ${ }^{15}$ We introduced into this largescale map three important additions: (i) Manually curated interactions between PCD proteins, which were missing from the public data sets (see our initial connectivity map in Supplementary Figure S2, and supplementary Table 2 for the full list of interactions between proteins in the PCD set). (ii) Adding edge directionality (i.e., identifying one protein as the source and the other as the target) which is mostly missing in the available database. We manually determined the directionality of these edges on the basis of the literature, and if the directionality was not clear, it was assumed that the edge is bidirectional. (iii) Adding confidence values to nodes and edges. We assigned each interaction a confidence value based on the amount of supporting experimental evidence, using a logistic regression model adapted from Sharan et al. ${ }^{16}$ (see supplementary information for the method used for adding the confidence values on the interactions between the proteins). The proteins in the network were also assigned a confidence level, reflecting their functional relatedness to proteins in the PCD set (supplementary Table 1). This functional relatedness was calculated on the basis of the gene ontology (GO) biological process annotations using 
a
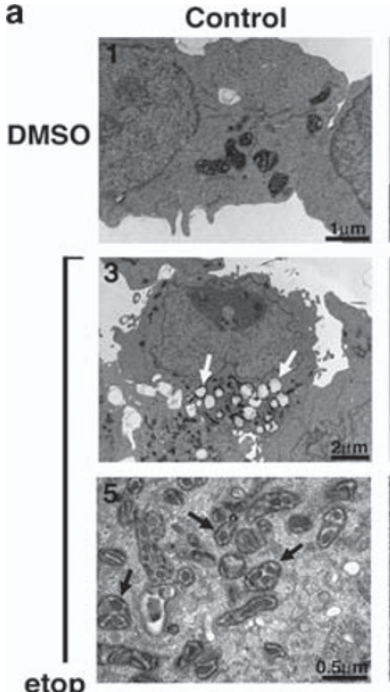

etop
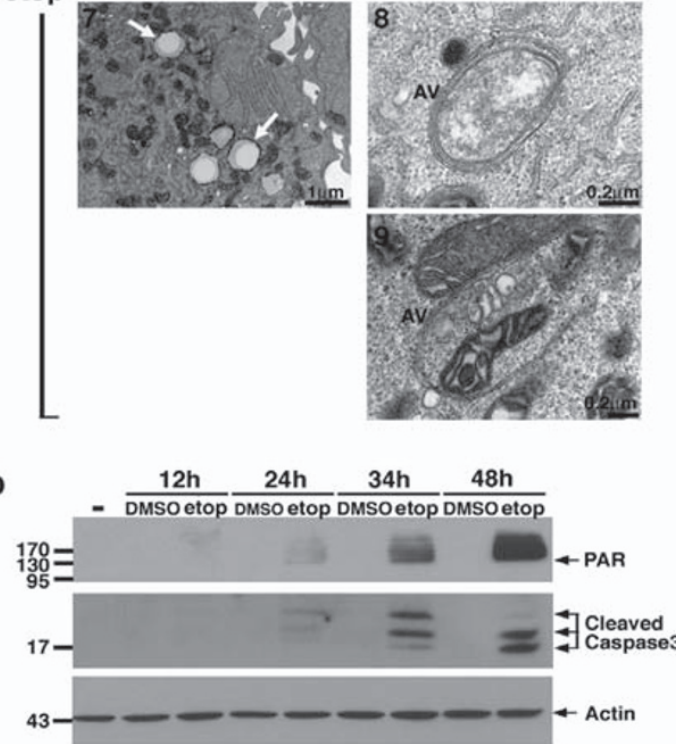

C

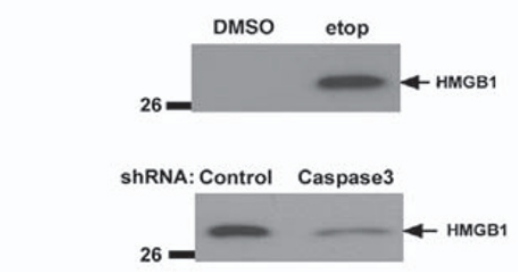

Caspase3 shRNA
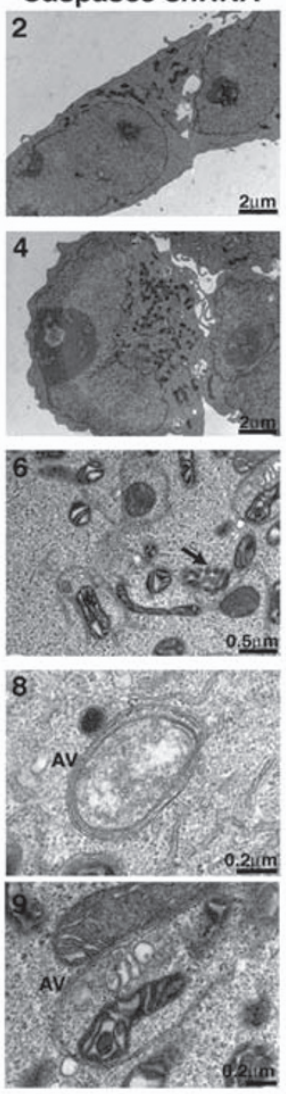

e

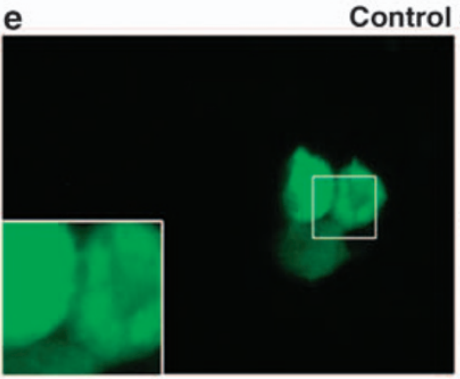

Caspase3 shRNA

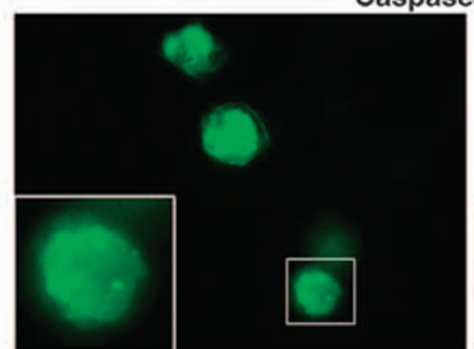

d

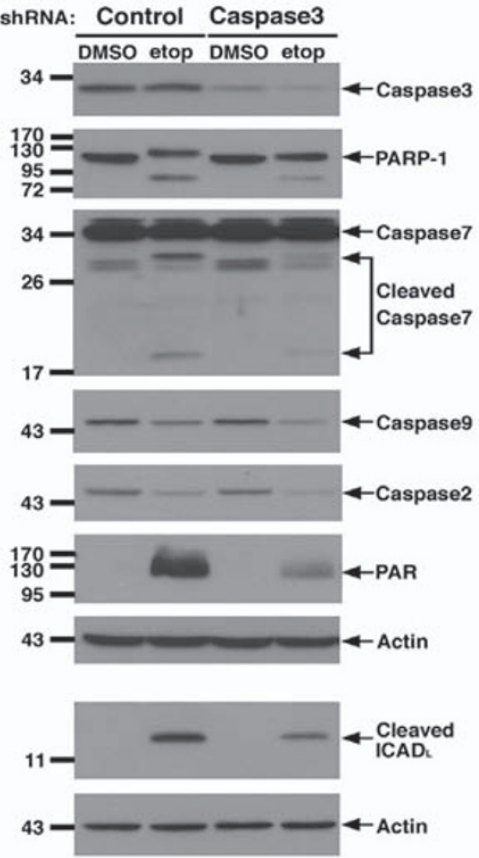

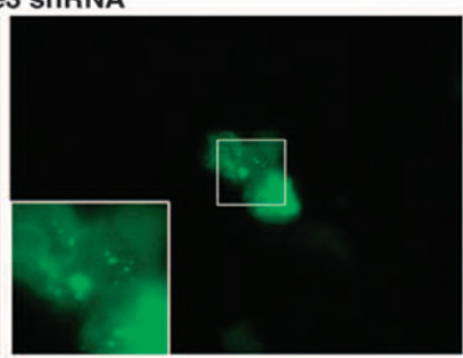

Figure 3 Caspase 3 perturbation changes the molecular signature and phenotypic hallmarks of etoposide-treated HEK293 cells. (a) Transmission electron micrographs of 293-Luc cells transfected with caspase3-targeting shRNA or with HcRed-targeting shRNA as a negative control, subjected to $48 \mathrm{~h}$ treatment with DMSO (a1 and a2) or $50 \mu \mathrm{M}$ etoposide (a3-a9). Figures a5-a9 were taken at higher magnification (see scale bar). White arrows indicate empty vacuoles. Black arrows point to severely damaged mitochondria. Notice that the number of severely damaged mitochondria was lower in the caspase3-perturbed cells (compare a5 with a6). 'AV' indicates autophagic vacuoles. (b) 293 cells were treated with $50 \mu \mathrm{M}$ etoposide or DMSO for the indicated time periods. The cells were then extracted, and western blot analysis was performed using the indicated antibodies. Note that the anti-PAR antibodies measure the overall PAR-ribosylation of cellular proteins. (-) represents non-treated cells at time 0 . (c) 293-Luc cells were transfected with shRNA targeting caspase 3 or with HcRed-targeting shRNA as a negative control, and were treated as in Figure 1 ( $9 \mathrm{~cm}$ plates). After $48 \mathrm{~h}$ etoposide treatment, the conditioned media were collected and concentrated, and in parallel, the cells were lysed using PLB buffer (for further information, see supplementary information). The media were normalized to the amount of extracted proteins from cells, and loaded on $12 \%$ gel. Western blot analysis was performed using anti-HMGB1 antibodies. Upper gel - HcRed control cells treated with etoposide or DMSO. Lower gel-HcRed control cells or caspase3 perturbed cells treated with etoposide. (d) 293-Luc cells were transfected with shRNA targeting caspase 3 or with HcRed-targeting shRNA as a negative control, and were treated as in Figure 1 ( $9 \mathrm{~cm}$ plates). Western blot analysis (upper panels $-12 \%$ gel, lower panels - $15 \%$ gel) was performed using the indicated antibodies. The molecular analysis shown here was repeated in more than three independent experiments. (e) Representative GFP-LC3 staining of 293-Luc cells transfected with caspase3 or the control shRNA, treated with $50 \mu \mathrm{M}$ etoposide for $48 \mathrm{~h}$ (for further information, see supplementary information) 


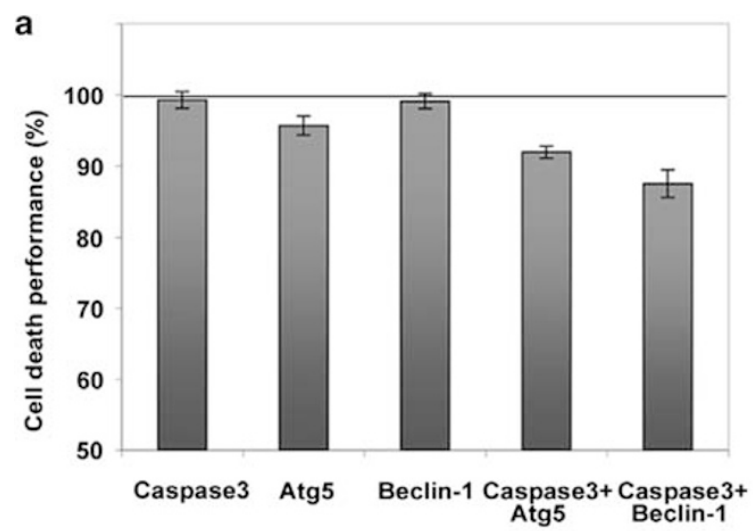

silencing perturbation

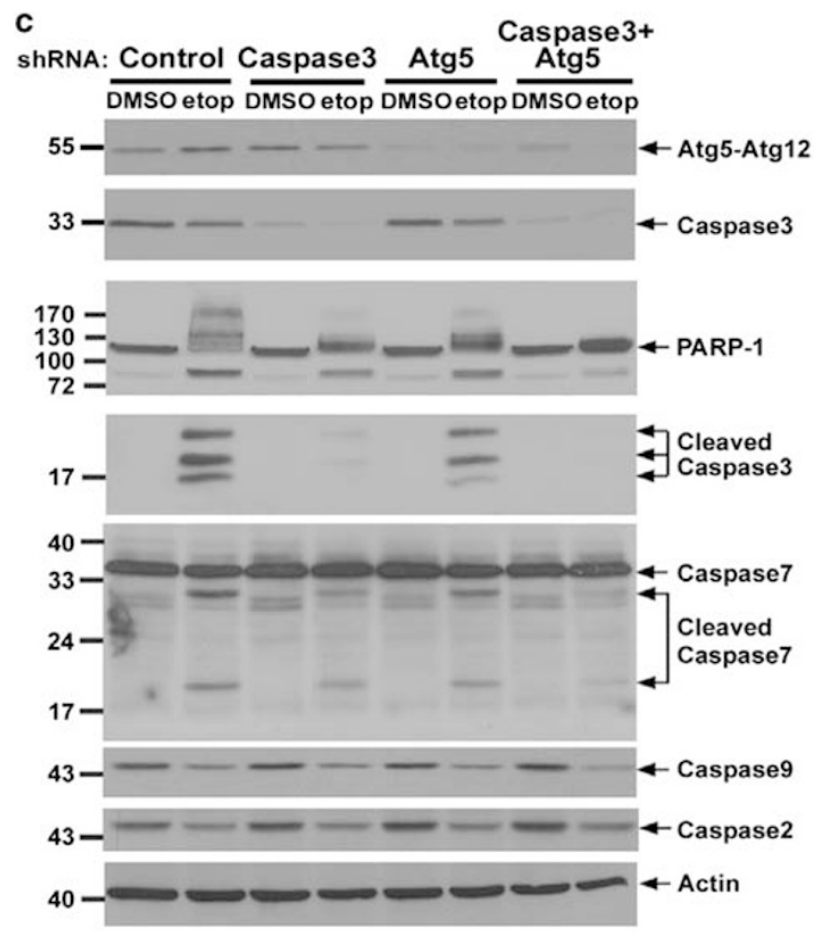

b

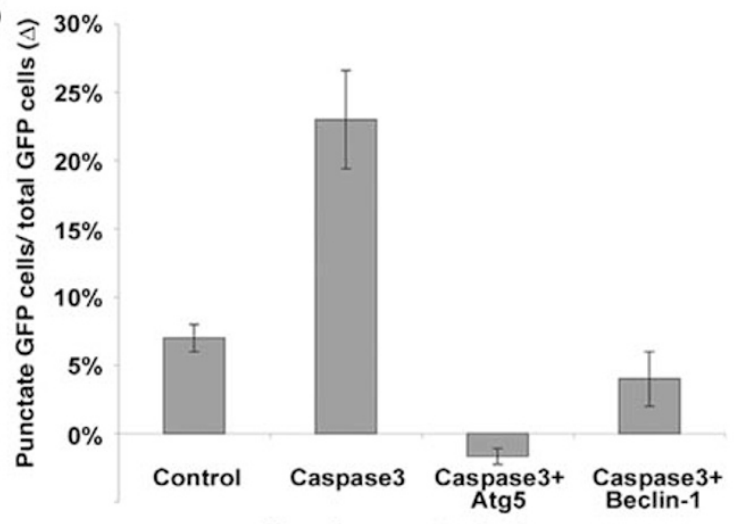

silencing perturbation

d shRNA: Control Caspase3 Beclin-1 Beclin-1

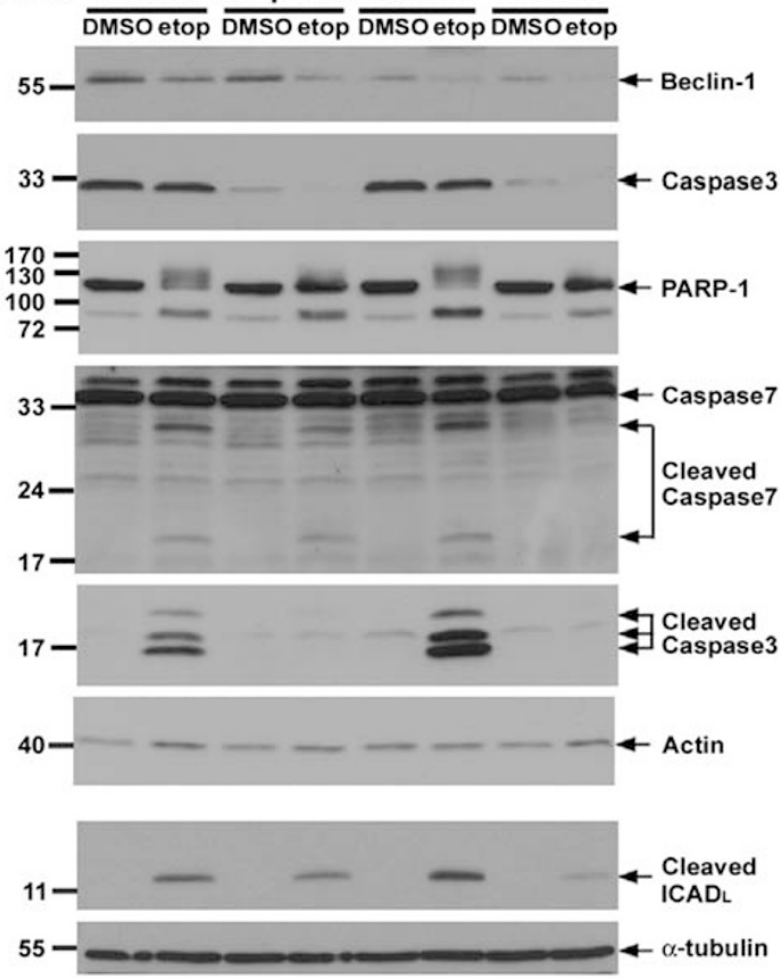

Figure 4 Knocking-down caspase3, Atg5 and Beclin-1 as single and double silencing perturbations. (a) Measurements of the cell death performance values of 293-Luc cells with the indicated silencing perturbations were performed as described in the Materials and Methods section. Data presented are the mean \pm S.D. of three independent experiments for Atg5, Beclin-1 and the combinatorial silencing perturbations, and of six independent experiments for the caspase 3 perturbation. The cell death values in the HcRed-transfected non-perturbed cells (mean from six independent experiments) were normalized to $100 \%$ cell death performance, and the cell death values of the single and double perturbations were modified into \% of cell death performance accordingly. The statistical processing of the data is detailed in the Materials and Methods section (b) Quantification of the percentage of cells with punctate GFP-LC3 fluorescence per total GFP-LC3-positive cells appearing after $48 \mathrm{~h}$ etoposide treatment in the single and double perturbations. Data presented are the mean \pm S.D. of the increase in punctate staining, after subtracting the basal levels in non-treated cells, in triplicate plates. The results were repeated in three independent experiments. (For further information, see supplementary information). (c and d) 293-Luc cells were transfected with the indicated shRNAs and treated as in Figure 1 ( $9 \mathrm{~cm}$ plates). Western blot analysis (d, lower panels $-15 \%$ gel, other panels $-12 \%$ gel) was performed using the indicated antibodies. The molecular analysis shown was repeated in more than three independent experiments. Note that in mammalian cells most of the Atg5 protein is already covalently linked to Atg12 under steady-state growth conditions (around $55 \mathrm{kDa}$ ). The ladder that is detected above the non-cleaved PARP-1 using the anti-PARP-1 antibody most probably represents auto-PAR ribosylation

semantic similarity ${ }^{17}$ (see Materials and Methods section). Using the augmented network, the most reliable pathways (i.e., having the highest overall confidence) connecting between a pair of proteins were identified (as detailed in Materials and Methods section), and this was then followed by experimental validation. 
Single perturbation experiments - knocking down caspase 3 reveals the existence of alternative caspase-independent mechanisms in the cell death network. Etoposide, a DNA-damaging drug, was chosen as the death-inducing agent in the current application of this platform. Phenotypic and molecular analysis indicated that a mixed type of necrotic and apoptotic cell death developed in the etoposide-treated HEK293 cells. Transmission electron microscopy (TEM) showed that the dying cells displayed hallmarks of programmed necrosis, including the appearance of empty vacuoles in the cytosol, and damaged mitochondria that were remodeled into separate matrix compartments (Figure 3a). Cells incorporated Sytox Green into their DNA indicative of loss of membrane integrity between 24 to $48 \mathrm{hrs}$ after etoposide treatment (Supplementary movie). Consistent with this phenotype, molecular analysis indicated that etoposide induced PAR ribosylation of proteins and the release of HMGB1 from the cells into the culture media (Figures $3 \mathrm{~b}$ and c), two previously characterized hallmarks of programmed necrosis. ${ }^{4,18-20}$ In addition, the canonical apoptotic markers developed in response to the drug, including activation of the upstream initiator caspases 2 and 9, and of the executioner caspases 3 and 7 and the cleavage of their two substrates, PARP-1 and ICAD (Figure $3 b$ and $d$ ). Also, fragmented nuclei and the release of cytochrome $c$ from mitochondria to the cytosol were frequently detected (Supplementary Figure S5). Time curve analysis showed that PAR-ribosylation and caspase 3 processing started to appear around $24 \mathrm{~h}$ after etoposide treatment, both peaking at $48 \mathrm{~h}$ (Figure $3 \mathrm{~b}$ ). Subsequent RNAi-mediated perturbations were all assessed $48 \mathrm{~h}$ after etoposide treatment, when the molecular responses were maximal.

Knocking down caspase3, a critical node of the apoptotic module (Figures 2, Supplementary Figure S2), resulted in reduced cleavage of the downstream substrates PARP-1 and $I C A D$, confirming that the apoptotic module downstream to caspase3 was attenuated (Figure $3 d$ ). Processing of caspase7, but not of caspases 9 and 2, was also reduced, indicating a positive interaction between the two executioner caspases, and excluding positive feedback loops with the upstream initiator caspases in this setting (Figure $3 d$ ). It is noted that the extent of etoposide-induced PAR ribosylation and HMGB1 release were also significantly attenuated by caspase 3 knock down (Figures $3 c$ and d), consistent with TEM studies that showed a reduction in the necrotic hallmarks (Figure 3a, panels 4, 6). In contrast, etoposide increased the appearance of autophagosomes in the caspase 3 knockeddown cells (Figure 3a, panels 8, 9). This was confirmed by scoring cells displaying punctate GFP-LC3 staining, a molecular marker for autophagosome formation. ${ }^{21}$ As shown in Figure $3 e$ and quantified in Figure $4 \mathrm{~b}$, the frequency of cells in which the GFP-LC3 appeared in puncta increased significantly upon exposure of caspase3-depleted cells to etoposide, compared with the non-perturbed cells. Thus, although the knock down of caspase 3 leads to the attenuation of the apoptotic and necrotic responses to etoposide, a switch to autophagy develops in parallel.

Surprisingly, measurement of cell death performance by the luciferase assay indicated a complete lack of death protection by caspase3 knock down (Figure 4a, Supplementary Figures S4b and S6a; note that two different shRNAs were used). The lack of death protection by caspase 3 knock down was also detected by the Trypan blue exclusion test (Supplementary Figure S3). It is noted that even when both caspases 3 and 7 were knocked-down simultaneously, no protection from cell death could be detected (Supplementary Figure S7), thus excluding the possibility that the residual cleavage of downstream proteins by caspase 7 might be responsible for the lack of cell death inhibition observed in the caspase 3 knockeddown cells. This prompted us to look at whether the switch to autophagy may have contributed to the observed caspaseindependent cell death, a possibility that which was further investigated by the epistatic analysis below.

Epistatic analysis - analyzing the genetic interactions between caspase 3 and either Atg 5 or Beclin-1 reveals additional levels of connectivity between the apoptotic and autophagic modules. To test whether the increased autophagy observed on caspase 3 knock down signifies a switch toward the autophagic cell death module, two essential components of the basic autophagic machinery, Atg5 and Beclin-1, were each knocked-down in combination with caspase 3 . Beclin-1 is part of a Class III PI3K complex, which functions at the nucleation step upstream to Atg5, which participates in membrane elongation of the growing autophagosome $^{22}$ (Figure 2 and Supplementary Figure S2).

The double perturbations were achieved by co-transfecting two shRNAs simultaneously. As shown in Figures $4 \mathrm{c}$ and d, the reduction of caspase 3 , Atg5 and Beclin-1 was similar irrespective of whether these genes were knocked-down independently or in combination with a second shRNA. Scoring GFP-LC3 puncta indicated that either Atg5 or Beclin-1 knock down completely suppressed the etoposideinduced increase in autophagy detected in caspase 3 perturbed cells (Figure 4b). Furthermore, although the single perturbation of Beclin-1 had no significant effect on cell death performance, the combination of Beclin-1 and caspase 3 depletion significantly reduced cell death performance value to $87.5 \%$ of its full capacity (Figure $4 a$ ). The calculated double perturbation fitness ${ }^{23}$ indicated a negative deviation from multiplicativity $(\varepsilon=-0.109$; see Materials and Methods section), that is, an aggravating type of interaction between Beclin-1 and caspase3. The latter is consistent with a model in which Beclin-1 is part of a backup death-promoting module activated when caspase 3 is knocked-down.

Unlike Beclin-1, the single perturbation of Atg5 displayed a small yet statistically significant reduction in cell death performance (Figure 4a, and Supplementary Figure S4b for raw data). In addition, the combination of Atg5 and caspase 3 knock downs reduced the cell death performance to $92 \%$ of its full capacity, yielding a nonsignificant deviation $(\varepsilon=-0.03)$ from the neutral multiplicative model. Thus, although both Beclin-1 and Atg5 are rate limiting for autophagy, the different outcomes in the double knock down analysis may suggest the existence of different modes of reciprocal connectivity to the apoptotic module, in addition to their canonical function in autophagy. Consistent with this possibility, we found that Atg5 knock down by itself attenuated the activation of caspases 3 and 7 by etoposide, resulting in a partial inhibition of PARP-1 cleavage (Figure 4c). A second shRNA targeting Atg5 
a

a

$$
\text { Atg5 } \rightarrow \text { RHPN2 } \rightarrow \text { KRT18 } \rightarrow \text { DEDD } \rightarrow \text { Caspase3 }
$$

b

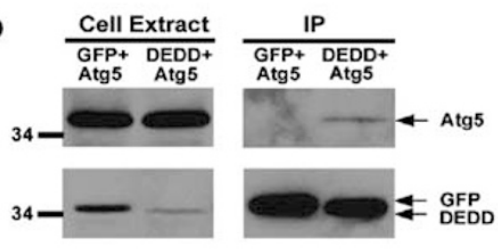

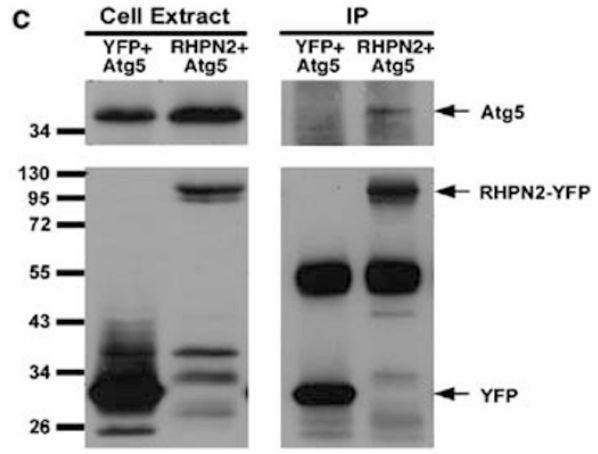

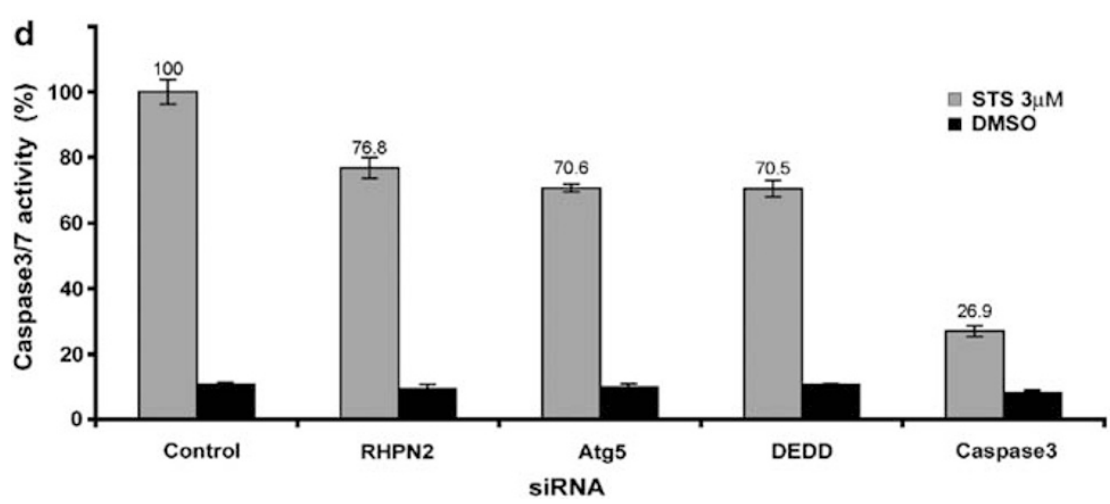

Figure 5 Exploring the new intermodular interaction between Atg5 and the apoptotic module using a computational approach. (a) A scheme describing the most reliable pathway found by the modified 'shortest path' algorithm that links Atg5 to caspase3. (b) HEK293-Luc cells were co-transfected with Flag-tagged DEDD or with Flag-tagged GFP together with HA-tagged Atg5. The Flag-tagged proteins were immunoprecipitated using anti-Flag antibodies, and the coimmunoprecipitated proteins as well as the total cell extracts were blotted with anti-Flag and anti-HA antibodies. (c) HEK293-Luc cells were co-transfected with YFP-tagged RHPN2 or with YFP alone together and HA-tagged Atg5. The YFP-tagged proteins were immunoprecipitated using anti-GFP antibodies, and the coimmunoprecipitated proteins as well as the total cell extracts were blotted with anti-GFP and anti-HA antibodies. (d) Measurements of caspase3/7 activity of HeLa cells treated with staurosporine with the indicated silencing perturbations were performed as described in the Materials and Methods section. Data presented are the mean \pm S.D. of four replicates. The values in the control cells treated with staurosporine were normalized to $100 \%$, and the caspase activity values of the DMSO-treated control, as well as the values of the different perturbations, were modified into \% of caspase activity accordingly. It is noted that the cell number of the DMSO-treated cells, determined by CyQUANT assay under the different perturbations, was similar

yielded the same results (Supplementary Figure S6b). Thus, Atg5 is positively connected to a pathway that activates caspase 3 , in addition to its canonical function in autophagy, thereby neutralizing the negative deviation from multiplicativity caused by the switch to autophagy. Beclin-1 on the other hand, is not connected in a positive manner to caspase3 activation; in contrast, it was found to be connected to the caspase-dependent pathway in a negative manner, resulting in a significant increase in caspase $3 / 7$ activation and in PARP-1 and ICAD cleavage on its knock down (Figure 4d, Supplementary Figure S6c). These conclusions further stress the power of combining fitness values with molecular data, for resolving the nature of epistatic interactions between pairs of genes.

\footnotetext{
Applying computational approaches to identify components of the pathway that potentially connects Atg5 to caspase3. Here, we further studied the newly discovered connectivity between caspase 3 and Atg5 in
}

response to etoposide. As we did not detect the presence of cleaved Atg5 (data not shown) in this setting, we assumed that the positive connection of Atg5 to apoptosis differs from previously described mechanisms. ${ }^{24,25}$ To translate the genetic interaction data into a molecular pathway, we applied a version of the shortest paths algorithm developed for this purpose, as described above, to identify the most reliable pathways. Several potential molecular pathways that link Atg5 to caspase 3 were identified by this algorithm; the highest scoring one was a pathway that links Atg5 to caspase3 through Rhophilin2 (RHPN2), cytokeratin18 and the death effector domain-containing DNAbinding protein (DEDD) (Figure 5a).

Cytokeratin 18 cleavage by caspase 3 was shown to be critical for the dismantling of epithelial cells during apoptosis. DEDD is a DED domain-containing protein, previously shown to be recruited to cytokeratin18 on induction of apoptosis, leading to caspase 3 activation and cytokeratin 18 cleavage. ${ }^{26}$ However, the mechanism of DEDD activation was not 
identified. RHPN2, also known as RhoB effector protein, is a PDZ-containing protein. The major function of PDZ-containing proteins is to act as scaffolds for the assembly of large protein complexes at specific subcellular locations, and to organize intracellular signaling. RHPN2 was found to interact with cytokeratin $18 ;{ }^{27}$ however, the functional implication of this interaction was not further examined. On the basis of this information, one possibility implies that RHPN2 might be needed to recruit Atg5 to cytokeratin18, resulting in Atg5DEDD interactions, to promote caspase 3 activity.

Experimentally, coimmunoprecipitation in HEK293-Luc transfectants revealed that HA-tagged Atg5 interacts with Flag-tagged DEDD, thus establishing a new connection between Atg5 and DEDD that was missing in the large-scale PPI data (Figure 5b). Also, these experiments further confirmed the interaction between HA-tagged Atg5 and YFP-tagged RHPN2 (Figure $5 \mathrm{c}$ ), previously suggested by the large-scale mass-spec screens. ${ }^{15}$

Next, the different components of this pathway (i.e., Atg5, DEDD, RHPN2) were knocked-down to assess their functional link to caspase 3 activation. To this end, HeLa cells, which are rich in Cytokeratin, ${ }^{18}$ were exposed to staurosporine, and caspase 3 activation was quantified by the CaspaseGlo luminescent assay. We found that in this cellular setting the knock down of Atg5 reduced caspase 3 activation, thus expanding the proapoptotic effects of Atg5 to other cell lines, and to another cell death-inducing agent (Figure 5d). Also, the knock down of DEDD reduced caspase 3 activation consistent with previous work. ${ }^{26}$ Moreover, the knock down of RHPN2 significantly reduced caspase 3 activation (Figure $5 d$ ), thus establishing for the first time its functional link to caspasedependent processes. These function-based assays, taken together with the co-immunoprecipitation experiments, are consistent with the suggestion raised by the computational approach, which links Atg5 to caspase3 activation through RHPN2 and DEDD. Thus, these data provide experimental confirmation to the computational predictions and document the potential power that resides in integrating the platform of RNAi-based perturbations with computational approaches, leading to the discovery of new biochemical pathways connecting between pairs of proteins.

\section{Discussion}

This work presents a multistage approach to analyzing the structure/function architecture of the molecular network underlying PCD. To this end, we developed a platform that measures the outcome of single and double RNAi-mediated perturbations on both the final cell death performance and the pattern of protein connectivity, and that uses computational methods for revealing the components of the pathways mediating the newly identified genetic interactions. We provide here a proof of principle that this approach is feasible, and show that it is possible to perform epistatic type of analysis in mammalian cells by using RNAi-mediated perturbations, similar to the genetic interaction maps performed in yeast by double mutation analysis. ${ }^{28-30}$ Specifically, the application of this platform to cells exposed to a DNAdamaging agent, in which critical nodes from the apoptotic and autophagic modules were targeted, enabled us to assess the extent to which the cross interactions between these functional modules affect the final cell death performance. Thus, intermodular connectivity provides an additional level of hierarchy in the global cell death network.

In addition, the precise quantifications by the luciferase assay were able to distinguish differences in the functional weights of different nodes in the same module. For example, we discovered that although the knock downs of Atg5 and Beclin-1 have similar effects on autophagy, they have different effects on cell death performance and on the apoptotic pathway, testifying to the additional different roles that they have in the network. This platform also provides leads for novel pathway discovery, an example of which was discovered here, linking an autophagic gene (Atg5) to the apoptotic module. Thus, the network analysis can be further translated into pathway discovery by combining the available PPI databases with the more precise knowledge on the PCD set of interactions and with advanced computational approaches applied here for this purpose.

Our detailed quantitative analysis indicates the existence of strong robustness in the PCD network that maintains the system performance even on attenuation of one or more death modules. Molecular analysis confirmed the existence of compensatory switches between modules, in which the activation of alternative death pathways acts as a backup mechanism. For example, the connectivity of caspase3 to autophagy allows an inducible and efficient switch from apoptosis to autophagic cell death on caspase3 perturbation, thus preventing a reduction in the overall cell death performance. The system presented here enables a quantitative analysis of the compensatory switch, as well as a means for the future identification of the critical molecular integrators that activate the switch.

The application of this platform should be scaled up to target hundreds of pairs of genes from the PCD map, with the intention of expanding this type of analysis to the structure of apoptotic/autophagic/necrotic modules, including the identification of various feedback loops and other complex intramodular circuits. Although the luciferase assay can be run in high-throughput format, most of the molecular assays require adaptation to multi well-based fluorescent/luminescent readouts, focusing on the critical positions along the pathways that signify activation of specific branches. In light of our finding that only an $8-14 \%$ reduction in cell death performance was achieved by the double perturbations of caspase3/Atg5 or caspase3/Beclin-1, it is conceivable that many additional backup mechanisms and compensatory switches may be activated in the global network of PCD, and remain to be identified in the future. Thus, collecting precise quantitative data provides a powerful tool for future unbiased genome-wide RNAi screens, which can be performed on cells already carrying well-characterized double perturbations. This will serve to select the additional perturbation(s) that lead to the complete collapse of cell death performance. (i.e., more than $90 \%$ reduction in cell death performance). Eventually, such high-throughput systems level analysis will reveal more basic principles of the hierarchical organization of the network, with the long-term goal of modeling the system and understanding both its structure and function. 


\section{Materials and Methods}

Generation of HEK293 cells stably expressing luciferase (293-Luc). To generate cells stably expressing luciferase, HEK293 cells were transfected with $\mathrm{pCDNA3}$ vector containing the firefly luciferase gene under the control of a CMV promoter. The cells were grown in the presence of $1 \mathrm{mg} / \mathrm{ml} \mathrm{G} 418$ (Calbiochem, San Diego, CA, USA), and selected clones were individually isolated to create monoclonal populations stably expressing luciferase. Seven clones were obtained, three of which expressed high levels of luciferase, while maintaining normal growth rates characteristic of the parental HEK293 cells, and similar responses to various death stimuli. One of these clones was chosen for further work.

\section{Luciferase-based cell viability assay and statistical} analysis. HEK293-Luc cells were transfected with shRNA targeting one or two genes, or with $\mathrm{HcRed}$-targeting shRNA as a negative control, by the standard calcium phosphate technique. Two days later, cells were seeded in parallel to black $96-w e l l$ plates with transparent bottoms (Nunc), and to $9 \mathrm{~cm}$ plates. After $24 \mathrm{~h}$, cells were treated with $50 \mu \mathrm{M}$ etoposide or DMSO (Sigma, Rehovot, Israel) as a negative control. At the indicated times, large plates were extracted for western blot analysis, whereas a luciferase assay was performed on 96-well plates, in 10 replicates for each perturbation (treated or non-treated). Cells were lysed in reporter lysis buffer (Promega, Madison, WI, USA), followed by a single freeze-thaw cycle. Cell viability was assessed by measuring luciferase activity (Promega), using the Veritas microplate luminometer (Turner BioSystems, Sunnyvale, CA, USA). Overall cell survival was determined by dividing the mean luciferase activity of cells treated with etoposide by the mean activity of cells treated with DMSO for each knock down experiment.

Caspase-Glo assay. HeLa JW cells were transfected with the indicated short interfering RNAi oligos (siRNAs) (Dharmacon, Termo Fisher Scientific, Lafayette, CO, USA), or with non-targeting siRNA as a negative control (non-targeting \#2, Dharmacon) by the standard calcium phosphate technique. Two days later, cells were seeded onto 96-well plates with transparent bottoms (Nunc). After $24 \mathrm{~h}$, cells were treated with $3 \mu \mathrm{M}$ staurosporine or DMSO (Sigma) for $4 \mathrm{~h}$ and subjected to caspase activity assay (CaspasGlo, Promega). The assay was performed in four replicates on 96-well plates using a microplate luminometer (Tumer BioSystems). Cell number was assessed using the CYQUANT assay (Invitrogen, Paisley, Scotland).

Statistical analysis. The numbers obtained by the luciferase-based assay, in 10 replicates for each perturbation (e.g., see Supplementary Figures S4b and S6a), were subjected to statistical analysis, and a difference from the non-perturbed cells was considered statistically significant if the resulting test statistic was higher than $2 /$ lower than -2 . As we are dealing with a ratio, its variance was calculated by the variance of the ratio equation as follows: ${ }^{31}$

$$
\operatorname{Var}\left(\frac{\bar{X}_{1}}{\bar{X}_{2}}\right)=\frac{\operatorname{Var}\left(X_{1}\right)}{\theta_{2}^{2}}+\frac{\theta_{1}^{2} \operatorname{Var}\left(X_{2}\right)}{\theta_{2}^{4}}
$$

Where Var stands for variance and $\theta_{i}, i=1,2$, is the mean of the distribution of $X_{i}$.

The test statistic is:

$$
t=\frac{\left(\frac{\bar{X}_{1}}{\bar{X}_{2}}\right)-\left(\frac{\bar{Y}_{1}}{Y_{2}}\right)}{\sqrt{\operatorname{Var}\left(\frac{\bar{X}_{1}}{X_{2}}\right)+\operatorname{Var}\left(\frac{\bar{Y}_{1}}{Y_{2}}\right)}}
$$

Where $X_{1,2}$ is the perturbed case with/ without etoposide, and $Y_{1,2}$ is the nonperturbed case with/ without etoposide.

Owing to biological variation, each experiment was repeated at least three times (each time in 10 replicates for each perturbation, as described above). The test statistic numbers obtained from three independent experiments for Atg5 single perturbation, or from the double perturbation experiments were subjected to Fisher's combined probability test. ${ }^{32}$ A difference was considered statistically significant if the resulting $P$-value was smaller than 0.05 . The single Atg5 perturbation, and the two pairs of double perturbations analyzed in this work, displayed statistically significant $P$-values.

The distribution of the test statistic numbers obtained from nine independent experiments for caspase3, and from three independent experiments for Beclin-1, were compared with the distribution obtained when the non-perturbed (control) values were compared with each other. Although the latter was lower than caspase3 and Beclin-1's distribution, the results were not conclusive. Moreover, S.D. calculation for the cell death performance values (see below) clearly showed that the cell death performance obtained when caspase 3 or Beclin-1 was perturbed was not statistically different from the control (Figure 4a). Therefore, we concluded that caspase 3 and Beclin-1 single perturbations did not influence the cell death performance obtained when 293-Luc cells were treated with etoposide.

The values reflecting the percentage cell death (derived from three independent experiments for Atg5, Beclin-1, and the combinatorial silencing perturbations, and of three or six independent experiments for the caspase3 perturbation) were normalized to the control non-perturbed cells to obtain the cell death performance value (i.e., $100 \%$ cell death performance is the maximum cell death capacity measured in the unperturbed cells). S.D. values for the average cell death performance were then calculated.

Epistasis test. The double perturbation fitness calculations were performed as follows:. The cell death performance measured under silencing perturbation of protein $X$ or protein $Y$ is defined as $W_{x}$ and $W_{y}$, respectively. The expected cell death performance of double perturbation of proteins that operate along independent pathways is $W_{x} \times W_{y}$, and the measured cell death performance is defined as $W_{x y}$. The deviation from multiplicativity (i.e., $\varepsilon$ ) was calculated as the difference between the cell death performance obtained experimentally and the calculated (expected) cell death performance (i.e., $\varepsilon_{x y}=W_{x y}-W_{x} \times W_{y}$ ). ${ }^{23}$

An aggravating interaction was considered statistically significant when the respective $\varepsilon$ was more than 1 S.D. away from 0 .

The S.D. of $\varepsilon(D \varepsilon)$ was calculated as:

$$
\mathrm{D} \varepsilon=\mathrm{D}\left(\mathrm{W}_{\mathrm{xy}}\right)+\mathrm{D}\left(\mathrm{W}_{\mathbf{x}} \times \mathrm{W}_{\mathrm{y}}\right)
$$

The S.D. of the expected performance $\left(D\left(W_{x} \times W_{y}\right)\right)$ was calculated using a formula of uncertainty as follows:

$$
\mathrm{D}\left(\mathrm{W}_{\mathrm{x}} \times \mathrm{W}_{\mathrm{y}}\right)=\mathrm{W}_{\mathrm{y}} \times \mathrm{DW}_{\mathrm{x}}+\mathrm{W}_{\mathrm{x}} \times \mathrm{DW}_{\mathrm{y}}
$$

Although the negative deviation from multiplicativity in the caspase3/Beclin1 double perturbations was statistically significant, the double perturbation fitness calculation of caspase3/Atg5 was not significantly different from 0 .

Pathway inference. We represent the human PPI network as a directed graph where nodes correspond to proteins and edges connect interacting proteins. Every interaction $e$ is assigned a confidence value $0<P(e)<1$, computed as in study by Sharan et al. ${ }^{16}$ Every protein $v$ is assigned a confidence value $0<P(v)<1$, defined as $\min _{u \in Q} \operatorname{ssim}(u, v)$, where $Q$ is the PCD protein set and $\operatorname{sim}(u, v)$ is the semantic similarity ${ }^{17}$ of the proteins $u$ and $v$, based on the GO biological process annotations.

Assuming independence between the proteins, the overall likelihood of a path $H$ is:

$$
p(H)=\prod_{e \in E(H)} p(e) \prod_{v \in V(H)} p(v)
$$

where $E(H)$, and $V(H)$ are the edges and nodes in $H$. To control for the length of the inferred paths, we follow the approach of Shachar et $a l^{33}$ and add a lengthpenalizing factor by redefining the probability of $H$ as:

$$
p(H)=e^{-\delta|E(H)|} \prod_{e \in E(H)} p(e) \prod_{v \in V(H)} p(v)
$$

where the per-edge penalty $e^{-\delta}$ is set to the probability of an edge at the 25th percentile.

To facilitate the computation of a reliable path between a given a pair of proteins, we define a weight function $w$ on the nodes and edges in the network. The weights are taken as the $-\log$ of the respective confidence value. We then search for a path $H$ along which the sum of weights of nodes and edges is minimum. An optimal solution for this problem can be found in polynomial time using the Dijkstra algorithm. In our implementation, we used an extension of this algorithm and considered all pathways whose weight is up to a factor of $5 \%$ from the optimal solution.

Supplementary methods. Cell culture, DNA constructs, shRNAs, siRNAs, GFP-LC3 punctate staining assay, HMGB1 release assay, Trypan blue assay, TEM, DAPI staining and cytochrome $c$ release assay, protein analysis and antibodies, coimmunoprecipitation, adding confidence values on the interactions between the proteins, and pseudocode of the extended shortest paths algorithm. 
Acknowledgements. We thank Helena Sabanay for her assistance in performing the TEM experiments, Sara Barak for performing the antiviral response assay, and Yulia Idelchuk for essential technical help. We thank Yitzhak Pilpel for fruitful discussions and for commenting on the manuscript, Edna Schechtman for help and advice in statistical analysis and Z Kam for the help in setting up the live imaging system. We thank N Mizushima and T Yoshimori for providing the GFP-LC3 plasmid, YK Jung for the Atg5-HA plasmid, ME Peter for the Flag-DEDD plasmid and $Y$ Lazebnik for providing anti-Caspase9 and anti-Caspase7 antibodies. This work was supported by the Kahn Fund for System Biology at the Weizmann Institute of Science and by the EU grant (APO-SYS) to A Kimchi. A Kimchi is the incumbent of the Helena Rubinstein Chair of Cancer Research. R Sharan and E Ruppin were supported by a converging technologies grant from the Israel Science Foundation.

\section{Conflict of interest}

The authors declare no conflict of interest.

1. Galluzzi L, Maiuri MC, Vitale I, Zischka H, Castedo M, Zitvogel L et al. Cell death modalities: classification and pathophysiological implications. Cell Death Differ 2007; 14: 1237-1243.

2. Shimizu S, Kanaseki T, Mizushima N, Mizuta T, Arakawa-Kobayashi S, Thompson CB et al. Role of Bcl-2 family proteins in a non-apoptotic programmed cell death dependent on autophagy genes. Nat Cell Biol 2004; 6: 1221-1228.

3. Yu L, Alva A, Su H, Dutt P, Freundt E, Welsh S et al. Regulation of an ATG7-beclin 1 program of autophagic cell death by caspase-8. Science 2004; 304: 1500-1502.

4. Zong WX, Ditsworth D, Bauer DE, Wang ZQ, Thompson CB. Alkylating DNA damage stimulates a regulated form of necrotic cell death. Genes Dev 2004; 18: 1272-1282.

5. Matsumura H, Shimizu Y, Ohsawa Y, Kawahara A, Uchiyama Y, Nagata S. Necrotic death pathway in Fas receptor signaling. J Cell Biol 2000; 151: 1247-1256.

6. Guillon-Munos A, van Bemmelen MX, Clarke PG. Role of phosphoinositide 3-kinase in the autophagic death of serum-deprived PC12 cells. Apoptosis 2005; 10: 1031-1041.

7. Maiuri MC, Zalckvar E, Kimchi A, Kroemer G. Self-eating and self-killing: crosstalk between autophagy and apoptosis. Nat Rev Mol Cell Biol 2007; 8: 741-752.

8. Chan EY, Kir S, Tooze SA. siRNA screening of the kinome identifies ULK1 as a multi-domain modulator of autophagy. J Biol Chem 2007; 282: 25464-25474.

9. Inbal B, Bialik S, Sabanay I, Shani G, Kimchi A. DAP kinase and DRP-1 mediate membrane blebbing and the formation of autophagic vesicles during programmed cell death. J Cell Biol 2002; 157: 455-468.

10. Tamm I, Wang Y, Sausville E, Scudiero DA, Vigna N, Oltersdorf T et al. IAP-family protein survivin inhibits caspase activity and apoptosis induced by Fas (CD95), Bax, caspases, and anticancer drugs. Cancer Res 1998; 58: 5315-5320.

11. Reef S, Zalckvar E, Shifman O, Bialik S, Sabanay H, Oren M et al. A short mitochondrial form of p19ARF induces autophagy and caspase-independent cell death. Mol Cell 2006; 22: 463-475.

12. Peri S, Navarro JD, Amanchy R, Kristiansen TZ, Jonnalagadda CK, Surendranath $V$ et al. Development of human protein reference database as an initial platform for approaching systems biology in humans. Genome Res 2003; 13: 2363-2371.

13. Rual JF, Venkatesan K, Hao T, Hirozane-Kishikawa T, Dricot A, Li N et al. Towards a proteome-scale map of the human protein-protein interaction network. Nature 2005; 437 1173-1178.
14. Stelzl U, Worm U, Lalowski M, Haenig C, Brembeck FH, Goehler $\mathrm{H}$ et al. A human protein-protein interaction network: a resource for annotating the proteome. Cell 2005; 122: 957-968.

15. Ewing RM, Chu P, Elisma F, Li H, Taylor P, Climie S et al. Large-scale mapping of human protein-protein interactions by mass spectrometry. Mol Syst Biol 2007; $3: 89$.

16. Sharan R, Suthram S, Kelley RM, Kuhn T, McCuine S, Uetz $P$ et al. Conserved patterns of protein interaction in multiple species. Proc Natl Acad Sci USA 2005; 102: 1974-1979.

17. Lord PW, Stevens RD, Brass A, Goble CA. Investigating semantic similarity measures across the Gene Ontology: the relationship between sequence and annotation. Bioinformatics 2003; 19: 1275-1283.

18. Moubarak RS, Yuste VJ, Artus C, Bouharrour A, Greer PA, Menissier-de Murcia J et al. Sequential activation of poly(ADP-ribose) polymerase 1, calpains, and Bax is essential in apoptosis-inducing factor-mediated programmed necrosis. Mol Cell Biol 2007; 27: 4844-4862.

19. Ditsworth D, Zong WX, Thompson CB. Activation of poly(ADP)-ribose polymerase (PARP-1) induces release of the pro-inflammatory mediator HMGB1 from the nucleus. J Biol Chem 2007; 282: 17845-17854.

20. Xu Y, Huang S, Liu ZG, Han J. Poly(ADP-ribose) polymerase-1 signaling to mitochondria in necrotic cell death requires RIP1/TRAF2-mediated JNK1 activation. J Biol Chem 2006; 281: 8788-8795.

21. Kabeya $\mathrm{Y}$, Mizushima N, Ueno T, Yamamoto A, Kirisako T, Noda T et al. LC3, a mammalian homologue of yeast Apg8p, is localized in autophagosome membranes after processing. EMBO J 2000; 19: 5720-5728.

22. Gozuacik D, Kimchi A. Autophagy and cell death. Curr Top Dev Biol 2007; 78: 217-245.

23. St Onge RP, Mani R, Oh J, Proctor M, Fung E, Davis RW et al. Systematic pathway analysis using high-resolution fitness profiling of combinatorial gene deletions. Nat Genet 2007; 39: 199-206.

24. Pyo JO, Jang MH, Kwon YK, Lee HJ, Jun JI, Woo HN et al. Essential roles of Atg 5 and FADD in autophagic cell death: dissection of autophagic cell death into vacuole formation and cell death. J Biol Chem 2005; 280: 20722-20729.

25. Yousefi S, Perozzo R, Schmid I, Ziemiecki A, Schaffner T, Scapozza L et al. Calpain-mediated cleavage of Atg5 switches autophagy to apoptosis. Nat Cell Biol 2006; 8: 1124-1132.

26. Lee JC, Schickling O, Stegh AH, Oshima RG, Dinsdale D, Cohen GM et al. DEDD regulates degradation of intermediate filaments during apoptosis. J Cell Biol 2002; 158: 1051-1066.

27. Mircescu H, Steuve S, Savonet V, Degraef C, Mellor H, Dumont JE et al. Identification and characterization of a novel activated RhoB binding protein containing a PDZ domain whose expression is specifically modulated in thyroid cells by cAMP. Eur J Biochem 2002; 269: 6241-6249.

28. Segre D, Deluna A, Church GM, Kishony R. Modular epistasis in yeast metabolism. Nat Genet 2005; 37: 77-83.

29. Tong AH, Lesage G, Bader GD, Ding $\mathrm{H}, \mathrm{Xu} \mathrm{H}$, Xin $\mathrm{X}$ et al. Global mapping of the yeast genetic interaction network. Science 2004; 303: 808-813.

30. Schuldiner M, Collins SR, Thompson NJ, Denic V, Bhamidipati A, Punna T et al. Exploration of the function and organization of the yeast early secretory pathway through an epistatic miniarray profile. Cell 2005; 123: 507-519.

31. Stuart A, Ord JK. Kendall's Advanced Theory of Statistics. 5 edn. Oxford University Press, Inc. New York, NY, USA, 1987; 1: pp.325.

32. Whitlock MC. Combining probability from independent tests: the weighted Z-method is superior to Fisher's approach. J Evol Biol 2005; 18: 1368-1373.

33. Shachar R, Ungar L, Kupiec M, Ruppin E, Sharan R. A systems-level approach to mapping the telomere length maintenance gene circuitry. Mol Syst Biol 2008; 4: 172.

Supplementary Information accompanies the paper on Cell Death and Differentiation website (http://www.nature.com/cdd) 